University of Wollongong

Research Online

Faculty of Informatics - Papers (Archive)

Faculty of Engineering and Information

Sciences

$1-1-2007$

\title{
Adaptive self-organisation of wireless ad-hoc control networks
}

Fazel Naghdy

University of Wollongong, fazel@uow.edu.au

Nathan Simiana

University of Wollongong

Follow this and additional works at: https://ro.uow.edu.au/infopapers

Part of the Physical Sciences and Mathematics Commons

\section{Recommended Citation}

Naghdy, Fazel and Simiana, Nathan: Adaptive self-organisation of wireless ad-hoc control networks 2007, 109-112.

https://ro.uow.edu.au/infopapers/896

Research Online is the open access institutional repository for the University of Wollongong. For further information contact the UOW Library: research-pubs@uow.edu.au 


\title{
Adaptive self-organisation of wireless ad-hoc control networks
}

\begin{abstract}
A novel concept called Wireless ad-hoc Control Networks (WACNets), exploring an ad-hoc approach to networked distributed control, has been under study for the last five years in the research group. Such systems represent a new stage in the evolution of distributed control and monitoring. The work carried out in developing an adaptive self-organisation algorithm for WACNet is reported. The algorithm deploys a distance measure technique while satisfying the rules and assumptions developed for WACNet framework. The effectiveness of the algorithm is verified through computer simulation under a number of given scenarios. The results obtained show that the algorithm effectively drives a network through different stages of its evolution.
\end{abstract}

\section{Keywords}

control, organisation, self, adaptive, wireless, ad, networks, hoc

\section{Disciplines}

Physical Sciences and Mathematics

\section{Publication Details}

F. Naghdy \& N. Simiana, "Adaptive self-organisation of wireless ad-hoc control networks," in IEEE International Conference on Signal Processing and Communication, 2007, pp. 109-112. 


\title{
ADAPTIVE SELF-ORGANISATION OF WIRELESS AD-HOC CONTROL NETWORKS
}

\author{
Fazel Naghdy, Nathan Simiana \\ School of Electrical, Computer and Telecommunications Engineering,
University of Wollongong, Australia
}

\begin{abstract}
A novel concept called Wireless ad-hoc Control Networks (WACNets), exploring an ad-hoc approach to networked distributed control, has been under study for the last five years in the research group. Such systems represent a new stage in the evolution of distributed control and monitoring. The work carried out in developing an adaptive self-organisation algorithm for WACNet is reported. The algorithm deploys a distance measure technique while satisfying the rules and assumptions developed for WACNet framework. The effectiveness of the algorithm is verified through computer simulation under a number of given scenarios. The results obtained show that the algorithm effectively drives a network through different stages of its evolution.
\end{abstract}

Index Terms - distributed control, ad-hoc networks, wireless, self-organization, IEEE1451, smart sensor

\section{INTRODUCTION}

There have been substantial developments in the field of control systems since the eighteenth century, where James Watt performed work on the speed control of steam engines [1]. This advancement has run in parallel with both theoretical developments in control theory and the fast technological development witnessed in the digital age towards implementation of effective control systems.

The advances made in computer networking have had significant impact on development of industrial controllers. These control systems were traditionally central and hierarchical where large centrally located mini-computers were connected directly to dumb $\mathrm{I} / \mathrm{O}$ racks through the $\mathrm{I} / \mathrm{O}$ ports of the computer. The first major change took place when a distributed architecture was adopted. In this configuration, a central computer supervised distributed direct digital controllers (DDC) with some local intelligence in the $\mathrm{I} / \mathrm{O}$ modules.

In the 1980's, network connectivity was introduced between the supervisory computer system and DDC through dial up link. The supervisory computer ran the proprietary vendor software. The dial up connection was replaced by Ethernet in the early 90's and various control modules in the DDC were linked up by vendor proprietary networks.

Around 1995, the proprietary software running on the supervisory control was replaced by browser-based front -end and more standard network tools such as LONWorks [2] and BACnet [3] providing connectivity among the DDC's.

A concept called Wireless ad-hoc Control Networks (WACNets), exploring an ad-hoc approach to networked distributed control, has been under study for the last five years in this research group [4], [5]. Such systems represent a new stage in the evolution of distributed control and monitoring.

The primary goal of the study is to develop a framework for organic, evolutionary and scalable integration of a large number of intelligent and heterogeneous nodes. Each node is envisaged to consist of sensing and/or actuation, local intelligence and control, data processing and communication components.

In this paper, the work carried out in developing an adaptive self-organisation algorithm for WACNet is reported. The algorithm deploys a distance measure technique [6] while satisfying the rules and assumptions developed for the framework. The effectiveness of the algorithm is verified through computer simulation under a number of given scenarios. The results obtained show that the algorithm effectively drives a network through different stages of its evolution.

The paper is organised as follows. Initially, an overview of the WACNet architecture will be provided. The experimental test-bed developed to study the concept will be then introduced. The proposed for selforganisation of WACNet will be then described and the results obtained so far will be presented.

\section{ARCHITECTURE OF WACNET}

The architecture of the proposed framework is based on IEEE 1451 compliant smart-sensor standards as it provides ability for self-identification, self-testing and adaptive calibration [7]. A combination of IEEE 1451 and Bluetooth standard implemented to provide several advanced features such as plug-n-play while maintaining minimum hardware overhead at the transducer nodes. The overall model of a node is illustrated in Fig. 1.

As illustrated, a STIM communicates with an NCAP module over a Bluetooth link. The TII protocol, which 
defines a form of communication between the STIM and NCAP, is implemented via Bluetooth. NCAP nodes are connected to the local monitor and /or control system. An operator can monitor or control the whole system in real time through the local monitor system on demand. The network model of WACNet is described in Fig. 2.

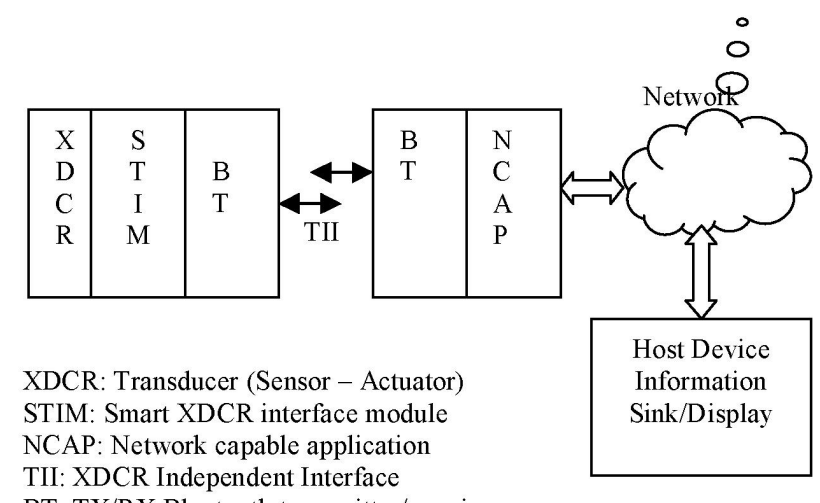

BT: TX/RX Bluetooth transmitter/ receiver

Fig. 1- WACNet architecture based on IEEE 1451 and Bluetooth standards

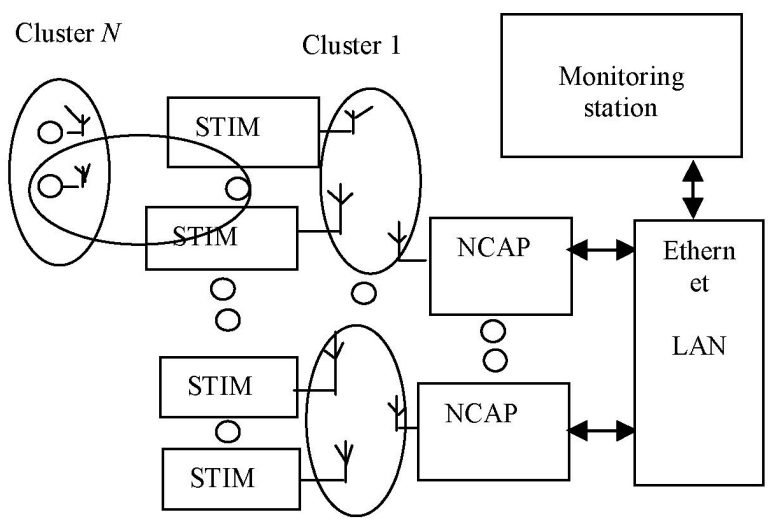

Fig. 2. The whole network model of WACNets

\section{EXPERIMENTAL TEST BED}

In order to validate the developed concepts, a test bed has been developed. In this system, the measured signal from the sensor is amplified, filtered and digitized via an A/D converter. The digitized data is then sent to a microcontroller unit (MCU) for processing. The digital signal produced in the node is converted into analogue before it is sent to drive the actuator. Sensors and actuators can be in different nodes. With the deployment of a Bluetooth (BT) wireless device, all data collected in the node can be transmitted wireless to other nodes.

The node in this work is illustrated in Fig. 3. The MCU is PIC16F877 which supports in-circuit programming, and allows the user's code to be downloaded into the PIC while it remains on the board. The Bluetooth system is implemented using ROK 101007 as it provides a reliable full duplex communication link, with the ability to form small networks called piconets, compliant with Bluetooth version $1.0 \mathrm{~B}$, low power consumption and has a range of 10 meters $(0 \mathrm{dBm})$. The golden coloured metal cover of ROK provides an excellent RF shedding. It also has a 50
OM antenna interface for the best signal strength performance.

The TINI Board [8], manufactured by Dallas Semiconductors, is deployed to construct the NCAP to transfer from the STIM nodes of a network and the monitoring stations associated with the network. Another Ericsson ROK 101007 Bluetooth Module provides communication between NCAP and the backbone network. The NCAP is illustrated in Fig. 4.

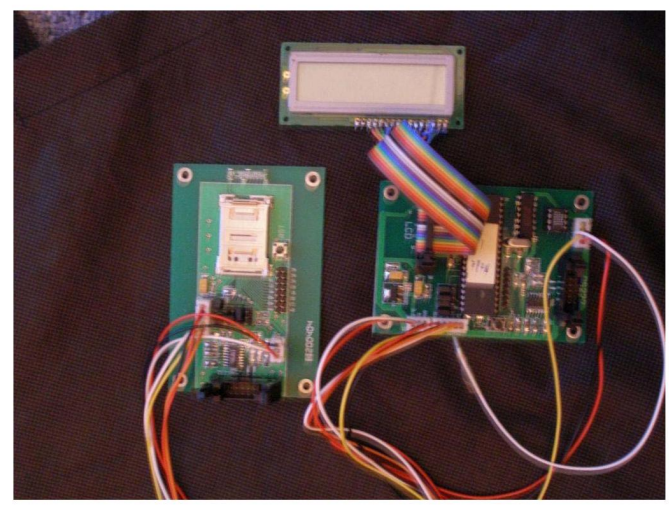

Fig. 3 STIM and Bluetooth Module

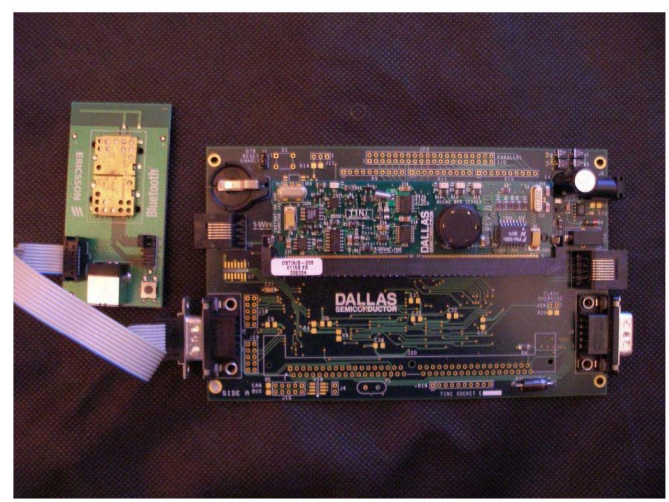

Fig. 4 NCAP Prototype Module

\section{SELF-ORGANISATION ALGORITHM}

The WACNet self-organisation algorithm is developed based on the following set of rules and assumptions [9]:

- A network is formed in clusters of STIM Nodes that communicate with NCAP Nodes;

- The STIM Nodes form clusters based on their ability to collaborate with each other to complete a common goal;

- Each cluster has a maximum of eight STIM Nodes;

- Each Cluster has a Master that is solely responsible for communication with the NCAP node in range. All slaves of the cluster relay information through the master node;

- Clusters that are out of range of an NCAP node can request a bridge with the master of another cluster that is within range of an NCAP Node; and

- All STIM Nodes of a cluster contain all relevant network information so that they can become a master in the event of a Node failure.

A task oriented approach is deployed in the development of the self-organisation method which does 
not rely on a node being aware of its geographical location [10]. The proposed algorithm uses a distance measure relating to a task that needs to be performed and the capability of a node attempting to determine its suitability to carry out the task. The attributes of a control task $(\mathrm{T})$ are broadcast by a node to all the nodes in a network, and each control node determines its suitability to perform the task based on two criteria [11].

The first criterion determines a distance value for a given parameter of a task $(\mathrm{T})$ with respect to the control node $(\mathrm{C})$ performing the calculation. This is illustrated in relationship (1) where $C$ is the control node, $T$ is the given task and Attr is an attribute relating to the task and the control node.

$$
d_{i}=1-\frac{C \cdot A t t r(i) \bigcap T . A t t r(i)}{C . A t t r(i) \bigcup T . A t t r(i)}
$$

The second index gives the sensor node $(\mathrm{C})$ an overall distance measure for all the task attributes calculated from (1). This is illustrated by (2) where $d_{i}$ is the distance measure for attribute $i$ and $n$ is the number of attributes tested.

$$
|d|=\sqrt{\frac{\sum_{i=1}^{n} d_{i}^{2}}{n}}
$$

In an ad-hoc network, the attributes of Task (T) is dynamically updated by the attributes of the cluster formed through self-organisation process.

The algorithm attempts to satisfy these rules through five major stages of STIM node master allocation; cluster formation; bridging STIM node master allocation, cluster consolidation; and cluster evolution.

Upon power up, a STIM node determines whether there is any master STIM Nodes within single hop communication range of itself. If there is a master STIM Node within range, the STIM Node will progress directly to the other four stages of cluster formation which include cluster formation, cluster consolidation, and cluster evolution.

\section{RESULTS}

The five stages of the algorithm have been validated through four test scenarios for a typical network as defined in Fig. 5. The test scenario 1 verified the functionality of stages 1, 2, 3 and 4 of the algorithm. After running the simulation, the following tasks were completed successfully for all nodes (Fig. 6):

- $\quad$ STIM Node Master Allocation;

- Cluster Formation;

- Bridging Master STIM Node Allocation; and

- Cluster Consolidation

The test scenario 2 verified the functionality of stage 5 of the algorithm in removing a master node (Fig. 7). After running the simulation application, the following tasks were completed successfully for all nodes of the given the network:

- Master STIM Node network detachment; and

- Cluster Re-Formation.

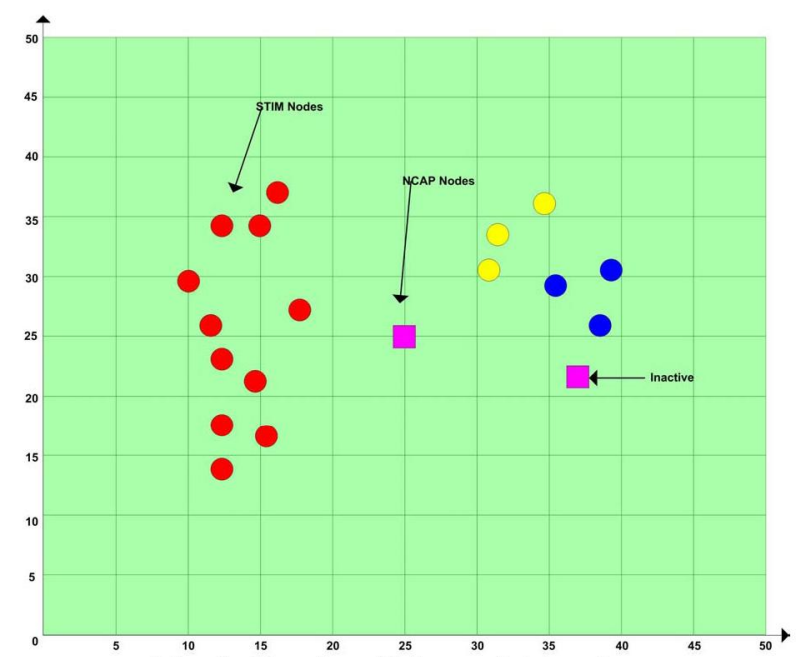

Fig.5, Simulated Network Topology

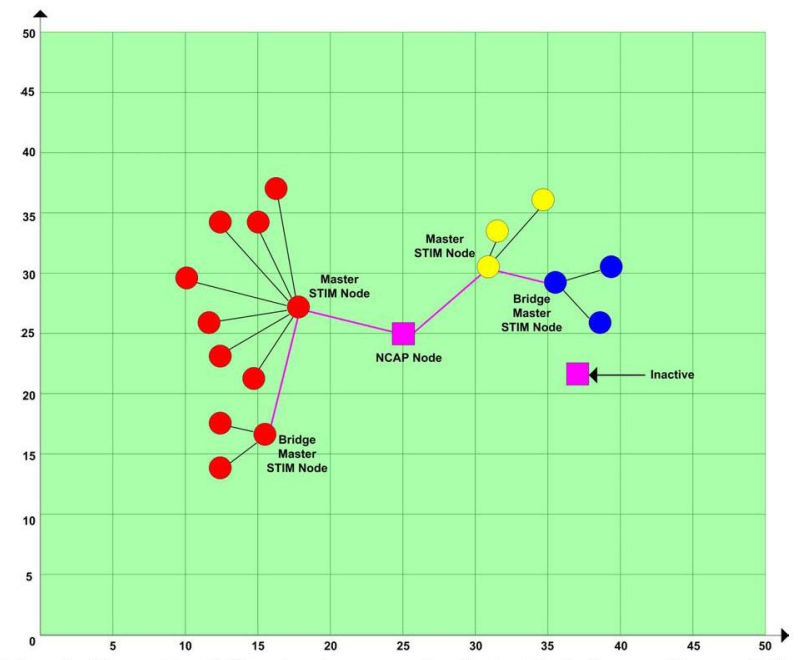

Fig 6. Result of the test scenario 1, initial formation of the network

Test Scenario 3 verified the functionality of Stage 5 of the algorithm in adding a NCAP node (Fig. 8). The simulation successfully validated the following tasks for all nodes:

- Addition of a NCAP Node to the network; and

- Cluster Re-Formation.

Finally, the test Scenario 4 verified the functionality of Stage 5 of the algorithms for the removal of an NCAP node (Fig. 9). The following tasks were completed successfully for all nodes:

- NCAP Node network detachment; and

- Cluster Re-Formation.

\section{CONCLUSION}

The work carried out on a new concept developed for the distributed control of real-time systems, called Wireless Ad-Hoc Control Networks (WACNet) was reported. The focus of the paper has been on a selforganisation algorithm which has been developed for autonomous operation and evolution of a WACNet.

During the paper the concept was introduced and the test-bed developed for its study was described. The core elements of the self-organisation algorithm were 
identified and its performance was validated through a series of studies carried out through computer simulation.

Observations show that the limitations of the algorithm relate mainly to the topology of the various nodes of a WACNet. These limitations should be overcome by developing the infrastructure required in supporting a WACNet.

The focus of the next stage of the project will be on implementing the algorithm on the WACNet test-bed and validate it for various real-time control scenarios.

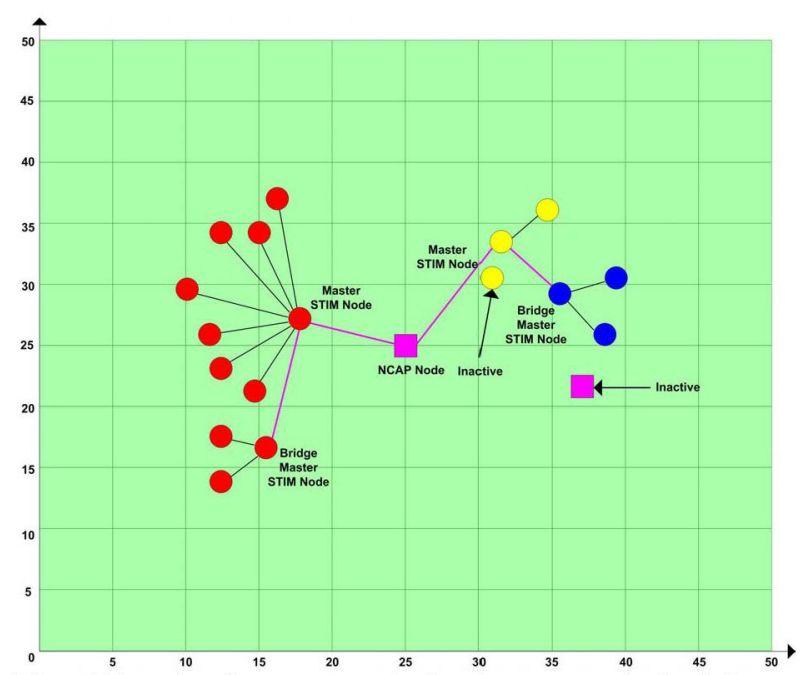

Fig. 7 Result of test scenario 2, the removal of a Master STIM Node from WACNet

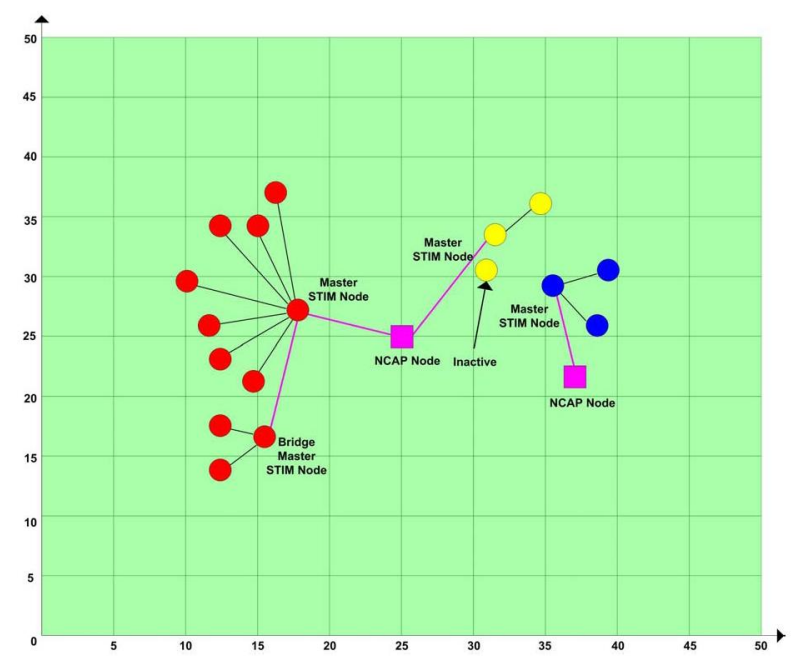

Fig. 8 Result of test scenario 3, addition of an NCAP Node

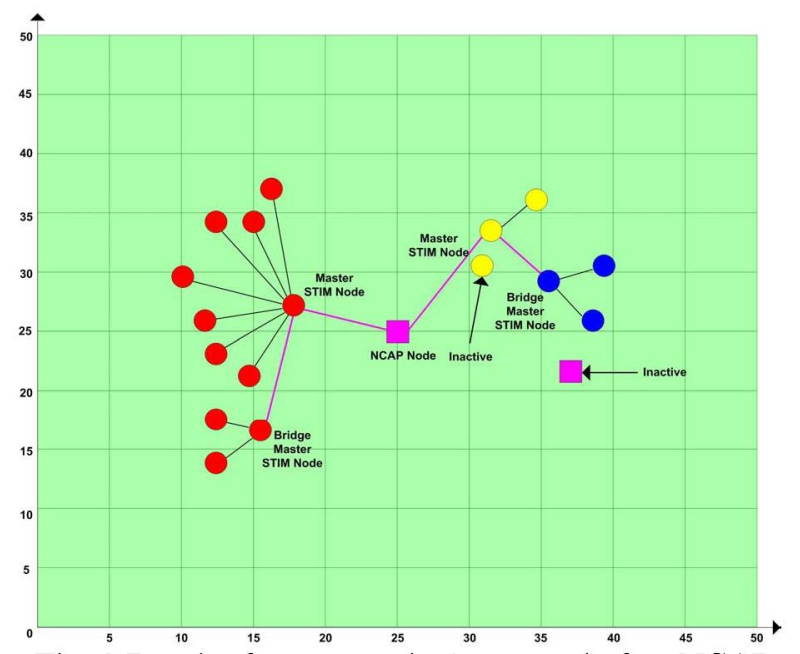

Fig. 9 Result of test scenario 4, removal of an NCAP Node

\section{REFERENCES}

[1] K. Ogata, Modern Control Engineering, 4th ed. Upper Saddle River, New Jersey: Prentice Hall, 2002.

[2] Full details are provided at http:/www.echelon.com/, [3]Full details are provided at http://www.bacnet.org/

[4] S. Bu, F. Naghdy, "Service Discovery in Wireless adhoc Control Networks, Proc. $2^{\text {nd }}$. International Conference on Intelligent Sensors, Sensor Networks and Information Processing - 2005 (ISSNIP 2005), Australia, $\mathrm{CD}$ publication.

[5] S. Bu, F. Naghdy, "Wireless ad-hoc Control Networks," Proc. 3rd International IEEE Conference on Industrial Informatics (INDIN), 2005, CD publication.

[6] J. Zhang, K. Premaratne and P. H. Bauer "A Distributed Self-Organization Algorithm for Ad-Hoc Sensor Networks," presented at IEEE Wireless Communications and Networking Conference, New Orleans, Louisiana, USA, 2003.

[7] Johnson, Robert N. "IEEE-1451.2 Update", http://www.sensorsmag.com/articles/0100/17/main.shtml. [8] Dallas Semiconductors, [TINI - Tiny InterNet Interfaces], [Online]. Available: http://www.maximic.com/TINIplatform.cfm.

[9] S. Bu, "Wireless Ad-Hoc Control Networks," ME (Research) thesis, School of Electrical, Computer and Telecommunications Engineering. Wollongong: University of Wollongong, 2005.

[10] Zhang, K. Premeratne, M. Dogruel and P. H. Bauer, "Task-Oriented Self-Organization of Ad Hoc Sensor Systems," presented at IEEE Sensors, 2002.

[11] J. Zhang, K. Premaratne and P. H. Bauer "A Distributed Self-Organization Algorithm for Ad-Hoc Sensor Networks," presented at IEEE Wireless Communications and Networking Conference, New Orleans, Louisiana, USA, 2003. 